\title{
Connexin mutations associated with palmoplantar keratoderma and profound deafness in a single family
}

\author{
David P Kelsell ${ }^{1}$, Amanda L Wilgoss ${ }^{1}$, Gabriela Richard ${ }^{2}$, Howard P Stevens ${ }^{1}$, \\ Colin S Munro ${ }^{3}$ and Irene M Leigh ${ }^{1}$
}

\begin{abstract}
${ }^{1}$ Centre for Cutaneous Research, St Bartholomew's and The Royal London School of Medicine and Dentistry, Queen Mary and Westfield College, London; ${ }^{2}$ Department of Dermatology and Cutaneous Biology, Thomas Jefferson University, Philadelphia, PA, USA; ${ }^{3}$ Department of Dermatology, Southern General Hospital, Glasgow, UK
\end{abstract}

\begin{abstract}
Recently, mutations in two gap junction genes, GJB2 and GJB3 (encoding Connexin 26 and Connexin 31 , respectively), have been shown to underlie either inherited hearing loss and skin disease or both disorders. In this study, we have extended our analysis of a small family in which palmoplantar keratoderma and various forms of deafness is segregating. In addition to the previously described sequence variant M $34 \mathrm{~T}$ in GJB2, two other sequence variants were identified: D66H also in GJB2 and R32W in GJB3. As D66H segregated with the skin disease, it is likely to underlie the palmoplantar keratoderma. The other two gap junction variants identified may contribute to the type of hearing impairment and the variable severity of the skin disease in the family. European Journal of Human Genetics (2000) 8, 141-144.
\end{abstract}

Keywords: Connexin; gap junction; keratoderma; deafness; mutation; polymorphism; Vohwinkel's syndrome

\section{Introduction}

Germline mutations in the gene encoding the gap junction protein Connexin 26 (GJB2) cause autosomal recessive deafness ${ }^{1}$ and, in some populations, underlie between $30-50 \%$ of genetic deafness. ${ }^{2,3}$ Although two reports have demonstrated that GJB2 mutations can also cause autosomal dominant deafness. ${ }^{4,5}$ it remains controversial as to whether the first described autosomal dominant deafness-associated variant, termed M34T, is actually disease-causing ${ }^{1}$ or just a low frequency non-consequential polymorphism. ${ }^{6}$ An in vitro assay for connexin channel activity suggests that M 34T is not a recessive or a neutral allele of GJB2 but acts as a dominant inhibitor of channel activity. ${ }^{7}$ However, the increasing reports of normal hearing M 34T carriers from distinct ethnic populations suggests that the inheritance of this sequence variant is not sufficient to cause hearing loss. To date, no M34T homozygotes have been reported. Here we provide a

Correspondence: Dr David P Kelsell, Centre for Cutaneous Research, St Bartholomew's and The Royal London School of Medicine and Dentistry, Queen Mary and Westfield College, 2 Newark Street, Whitechapel, London E1 2AT, UK. E-mail: kelsell@icrf.icnet.uk. Received 13 July 1999; revised 24 August 1999; accepted 2 September 1999 possible molecular explanation for the association of M34T with profound deafness.

The profound deafness-associated $M 34 T^{1}$ was identified in a small family in which palmoplantar keratoderma (PPK) and deafness was segregating. The PPK is diffuse and its distribution resembles that of a mild form of Vohwinkel's syndrome. ${ }^{8}$ The skin disease in individual II:2 differed from that seen in other members of the family in that there was a more extensive involvement of the palmoplantar skin. The nonvolar epidermis of this individual was also affected (data not shown). M34T was found in the DNA of two sisters (individualsII:2 and II:5; Figure1) who presented with the skin disease and profound hearing loss from birth. DNA was available from only one other family member; the daughter of one of the sisters (individual III:1) who was shown not to carry the M34T variant. As III:1 had the skin disease but not the profound form of hearing loss, this suggested that the M34T variant was associated with the profound deafness occurring in the family.

One explanation for M34T as being more significant than a neutral polymorphism is that its expressivity is dependent on its genetic background. However, no second-site mutations in GJB2 which may nullify the putative dominant effect 
of M34T in normal hearing carriers have been reported. Conversely, it is possible that M34T only causes genetic deafness when associated with a second mutation in either GJB2 or in another gene associated with hearing loss, for example, the gene encoding another gap junction protein, Connexin $\left.31(G) B 3:^{9}\right)$. Autosomal dominant mutations in GJB3 have also been associated with the skin disease, Erythrokeratoderma variabilis. ${ }^{10}$ In addition, the mitochondrial A7445G mutation has also been associated with both inherited deafness and a palmoplantar keratoderma. ${ }^{11}$

To investigate the latter possibility, DNA from three members of the PPK/deafness family in which M34T is segregating were reassessed for a second-site mutation in GJB2 and also for mutations in GJB3. Previously, none of the three family members were shown to carry the mitochondrial A7445G mutation (data not shown).

\section{Materials and methods Family material}

The clinical features of the palmoplantar keratoderma and the different types of hearing impairments (including audiograms) segregating in the family have been described previously. ${ }^{1,8}$ The structure of the family is shown in Figurelc. DNA was available from only three members of the family (individuals: II:2, II:5 and III:1). Genomic DNA extracted from 122 unrelated individuals was used as a control population.

\section{Mutation analysis}

The entire protein encoding region of both genes was amplified by PCR. GJB2 was performed as described previously. ${ }^{1}$ For GJB3, the coding sequence of human connexin 31 (GJB3) was amplified by PCR using the primer pair $5^{\prime}$ GTCAGAACTCAGAACACTGCC 3" corresponding to bases 681-701 and 5' CCTATACCCGGCTAGACAGC 3" corresponding to bases 1787-1768 (Genbank AJ004856) under the following PCR conditions: 35 cycles of $30 \mathrm{~s}$ at $94^{\circ} \mathrm{C}, 30 \mathrm{~s}$ at $62^{\circ} \mathrm{C}$ and $30 \mathrm{~s}$ at $72^{\circ} \mathrm{C}$. Each PCR fragment was screened for sequence variants by heteroduplex analysis and Denaturing High Performance Liquid Chromatography (DHPLC). All PCR reactions were heated to $95^{\circ} \mathrm{C}$ for $5 \mathrm{~min}$ followed by cooling to a final temperature of $25^{\circ} \mathrm{C}$. The samples were then transferred to the autosampler block, maintained at $7^{\circ} \mathrm{C}$, for analysis. DHPLC was performed using a WAVE $^{\mathrm{TM}}$ DNA Fragment analysis system (Transgenomic, Crew, Cheshire, UK). Following DHPLC, each variant PCR product was directly sequenced by PCR-cycle sequencing (Big-Dye terminator system, PE Applied Biosystems, Warrington, Cheshire, UK) and analysed on an ABI 377 automated sequencer (PE Applied Biosystems, Warrington, Cheshire, UK). The two further mutations identified, D66H in GJB2 and R32W in GJB3, were confirmed and used to screen their presence in the control population by restriction digestions using the restriction enzymes Dpnll and Hpall, respectively, Cloning of the
GJB2 PCR products were performed using the pGEM-T Easy Vector system (Promega, Southampton, UK) according to the manufacturer's instructions.

\section{Results}

Heteroduplex and DHPLC analysis revealed three variants in GJB2 and GJB3 in the family (Figurela). Sequencing identified the two new coding sequence variants, D66H in GJB2 and R32W in GJB3 (Figurelb). Restriction enzyme digests also confirmed the presence of these sequence variants (data not shown).

\section{Discussion}

In addition to identifying the previously described M34T variant in $11: 2$ and II:5, a second, previously undetected, sequence variant in GJB2, was present in all three individuals (Figure 1). This heterozygous G-C substitution in codon 66 results in a non-conservative amino acid change from an aspartic acid (GAT) to a histidine (CAT) (D66H) in the translated sequence. This sequence variant was not observed in 244 unrelated chromosomes and has not been reported in the literature as a polymorphism. The D66H carrier status was confirmed by restriction enzyme digestion of the PCR products as it results in the disruption of a DpnII site (data not shown). This aspartic acid residue is present in the first extracellular loop of GJB2 and is conserved in both beta and alpha types of connexins across different species, suggesting it is important for connexin activity. As all three individuals had the skin disease but not the profound deafness, it is likely that $\mathrm{D} 66 \mathrm{H}$ underlies the skin disease in the family (Figure 1c). From heteroduplex and DHPLC analysis plus sequencing of clones derived from the PCR products, M34T and D66H were shown to represent different alleles of GJB2.

A sequence variant in GJB3 was also identified in this family (Figure1). A single nucleotide substitution resulted in another non-conservative amino acid change from an arginine (CGG) to a tryptophan (TGG) in codon 32 of the translated sequence (R32W). The substitution disrupted a Hpall restriction site to allow independent confirmation of carrier status (data not shown). The arginine residue is present in the first transmembrane domain of the protein and is conserved in all connexin species. The R32W was present in individuals II:2 and III:1. Due to the association of GJB3 mutations and bilateral high-frequency hearing impairment, ${ }^{9}$ it is possible that R32W may contribute to the high frequency hearing impairment found in individual III:1. The panel of 244 unrelated chromosomes previously screened for the presence of $\mathrm{D} 66 \mathrm{H}$ were also assessed for R32W. One individual with no obvious cutaneous disorder or hearing impairment was heterozygous for R32W. The high frequency deafness may result from defective $C \times 31 / C \times 26$ channel formation and regulation when R32W in GJB3 is associated with $\mathrm{D} 66 \mathrm{H}$ in GJB2. However, it is equally plausible that $\mathrm{D} 66 \mathrm{H}$ in GJB2 underlies both the cutaneous disorder and the 

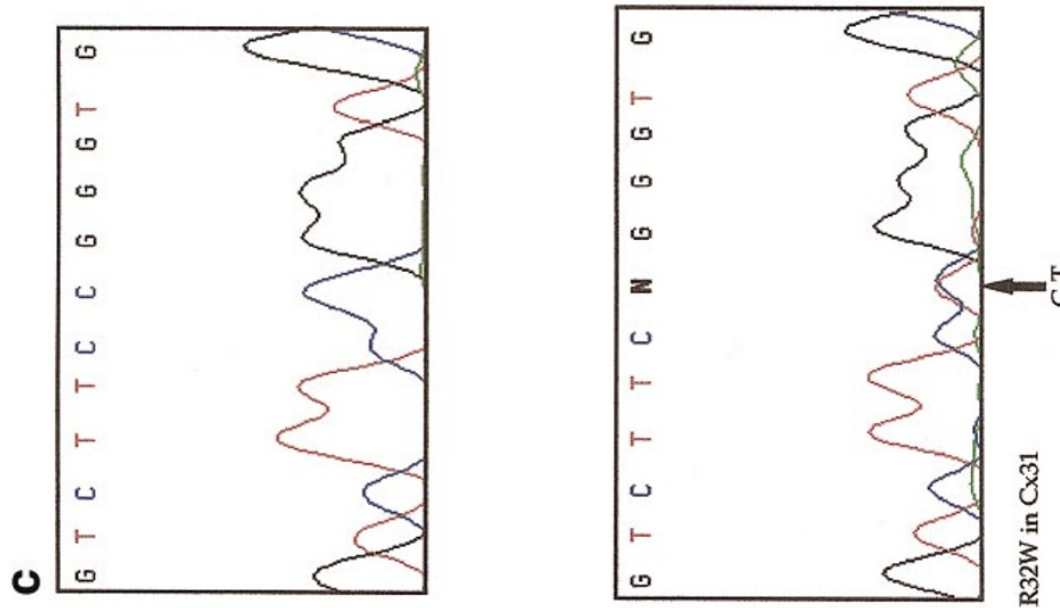

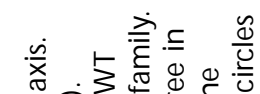

$\sigma \times \frac{\dot{x}}{\mathbf{x}}$

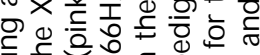

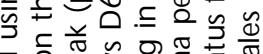

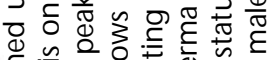

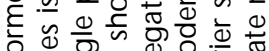

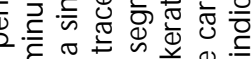

元

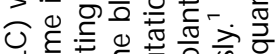

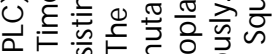

글 ¿ें

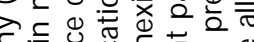

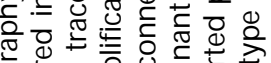

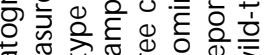

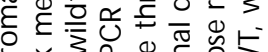

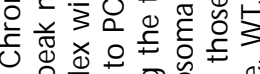
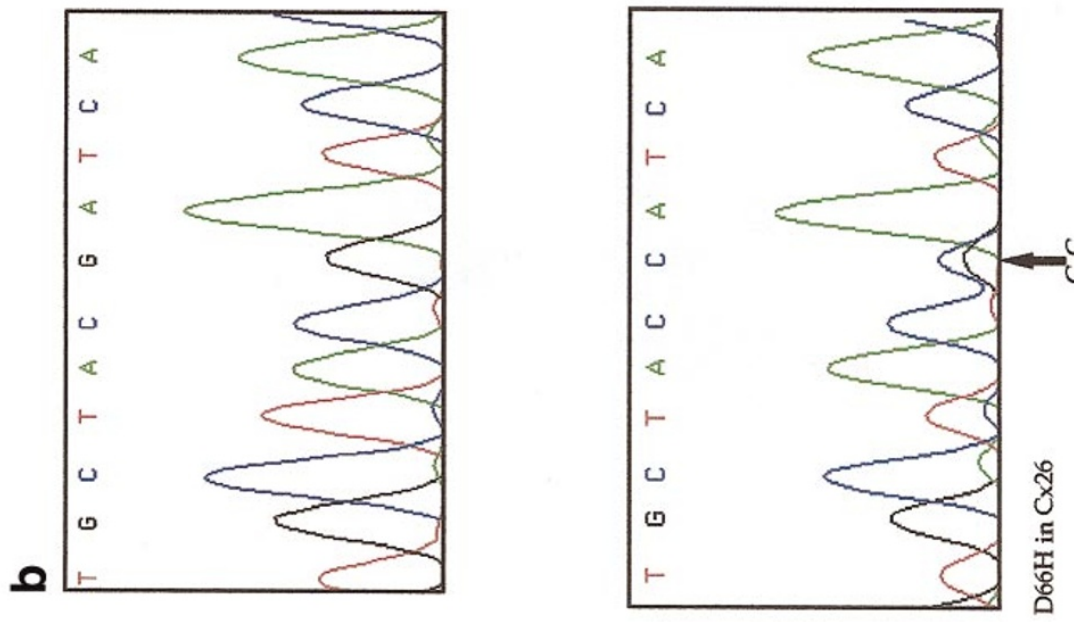

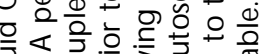

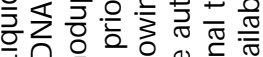

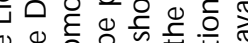

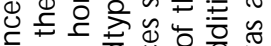

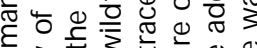

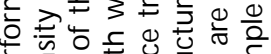

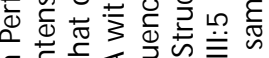

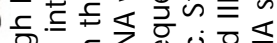

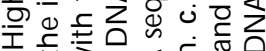

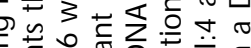

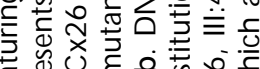

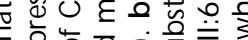

过 원

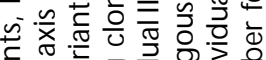

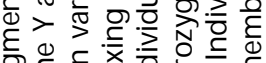

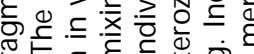

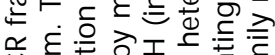

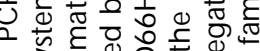

(1) है

는

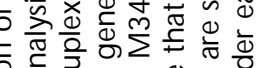

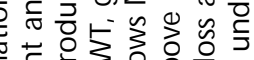

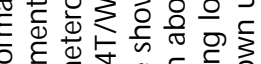

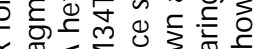

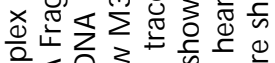

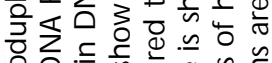

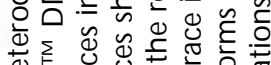

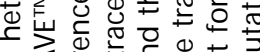

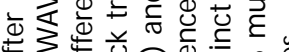

 
high frequency hearing loss in individual III:1. It is also possible that another mutation at a different locus, for example, that inherited from her deaf father (II:1), may contribute to the deafness in III:1.

As all tested individuals with PKK have only the D66H mutation in GJB2 in common, this suggests that this mutation primarily underlies the cutaneous phenotype in the family. In strong support of this is the recent identification of $\mathrm{D} 66 \mathrm{H}$ segregating with Vohwinkel's syndrome in three families, one of which was large. ${ }^{12}$ In these families, the individuals affected with the mutilating keratoderma presented with mild progressive hearing loss. None of the affected individuals from the larger of the families were carriers of M34T or R32W (data not shown). These findings would suggest that M34T does underlie the profound deafness in the family (Figure1c) but only on the genetic background of the second mutation in GJB2, D66H. Whether it acts as a recessive or modifying allele in combination with $\mathrm{D} 66 \mathrm{H}$ to cause profound deafness remains to be elucidated. In addition, M34T in GJB2 and R32W in GJB3 may also contribute to the more extensive manifestation of the skin disease which occurs in individual II:2.

\section{Acknowledgements}

This project was supported by the Imperial Cancer Research Fund. We thank Drs Liz Rugg, Tom W hite and David Paul for discussion.

\section{References}

1 Kelsell DP et al: Connexin 26 mutations in hereditary nonsyndromic sensorineural deafness. Nature 1997; 387: 80-83.

2 Zelante $L$ et al: Connexin 26 mutations associated with the most common form of non-syndromic neurosensory autosomal recessive deafness (DFNB1) in Mediterraneans. Hum Mol Genet 1997; 6: 1605-1609.

3 Denoyelle F et al: Prelingual deafness: high prevalence of a 30delG mutation in the connexin 26 gene. Hum Mol Genet 1997; 6: 2173-2177.

4 Denoyelle $F$ et al: Connexin 26 gene linked to a dominant deafness. Nature 1998; 393: 319-320.

5 Richard $G$ et al: Functional defects of $C \times 26$ resulting from a heterozygous missense mutation in a family with dominant deafmutism and palmoplantar keratoderma. Hum Genet 1998; 103: 393-399.

6 Scott DA, Kraft ML, Stone EM, Sheffield VC, Smith RJ: Connexin mutations and hearing loss. Nature 1998; 391: 32.

7 White TW, Deans MR, Kelsell DP, Paul DL: Connexin mutations in deafness. Nature 1998; 394: 630-631.

8 Fitzgerald DA, Verbov JL: Hereditary palmoplantar keratoderma with deafness. Br J Dermatol 1996; 134: 939-942.

$9 \mathrm{Xia} \mathrm{JH}$ et al: Mutations in the gene encoding gap junction protein beta-3 associated with autosomal dominant hearing impairment. Nat Genet 1998; 20: 370-373.

10 Richard $G$ et al: Mutations in the human connexin gene GJB3 cause erythrokeratodermia variabilis. Nat Genet 1998; 20: 366-369.

11 Sevior KB et al: Mitochondrial A7445G mutation in two pedigrees with palmoplantar keratoderma and deafness. Am J Med Genet 1998; 75: 179-185.

12 Maestrini $E$ et al: A missense mutation in connexin 26, D66H, causes mutiliating keratoderma with sensorineural deafness (Vohwinkel's syndrome) in three unrelated families. Hum Mol Genet 1999; 8: 1237-1243. 